\title{
Intra Subject Variability of Progesterone 200 mg Soft Capsules in Indian Healthy Adult Postmenopausal Female Subjects under Fasting Conditions
}

Rajeswara Rao P and Someswara Rao $K^{*}$

Andhra University College of Pharmaceutical Sciences, Andhra University, Visakhapatnam

\begin{abstract}
The aim of this study was to evaluate the intra subject variability of progesterone $200 \mathrm{mg}$ soft capsules of Test product with Prometrium ${ }^{\circledR}$ (Progesterone USP) capsules 200 mg (Reference) marketed by Solvay Pharmaceuticals Inc., Marietta, GA in healthy adult, human, post-menopausal female volunteers. This study was an open label, randomized, balanced, single-dose, two sequence two period, crossover oral bioequivalence study was conducted in 12 healthy adult, human, post-menopausal female volunteers under fasting conditions. Subjects received progesterone $200 \mathrm{mg}$ of either test or reference formulation with a washout period of 10 days. After study drug administration, serial blood samples were collected over a period of 36 hours post dose. The plasma concentrations of progesterone were determined by a validated method using LC/MS/MS. Pharmacokinetic parameters $C_{\max }, T_{\text {max }}$, $A \cup C_{0 . t}, A \cup C 0-\infty, K e l$ and $T_{1 / 2}$ were determined for both the formulations. The formulations were to be considered bioequivalent if the geometric least square mean ratio of test and reference of $C_{m a x}, A \cup C_{0-t}$ and $A U C_{0-\infty}$ were within the predetermined bioequivalence range of $80 \%$ to $125 \%$. A total of 12 subjects were enrolled. No significant differences were found based on analysis of variance. The $90 \%$ confidence intervals $(\mathrm{Cl})$ of $\mathrm{C}_{\max }, \mathrm{AUC}_{0-\mathrm{t}}$ and $\mathrm{AUC} \mathrm{C}_{0-\infty}$, of progesterone were $52.10-148.80 \%, 52.66-164.84 \%$, and $56.05-152.68 \%$ respectively. The test formulation in this study fails to show the bioequivalence with that of reference formulation for progesterone. The intra subject variability (\%) for $\mathrm{C}_{\text {max }}, \mathrm{AUC}_{0-\mathrm{t}}$ and $\mathrm{AUC}_{0-\infty}$ were found to be $68.2,75.6$ and 64.6 respectively. There was significant intra subject variability was observed for progesterone under fed conditions.
\end{abstract}

Keywords: Intra-subject variability; Highly variable drugs; Progesterone; Bioequivalence

\section{Introduction}

Bioequivalence (BE) studies are an integral component of the new drug development process. Additionally, they are required for the approval and marketing of generic drug products. BE studies are generally designed to determine if there is a significant difference in the rate and extent to which the active drug ingredient, or active moiety, becomes available at the site of drug action. According to the criteria developed by the U.S. Food and Drug Administration (FDA) and generally applied by other regulatory agencies, two pharmaceutically equivalent products are judged bioequivalent if the $90 \%$ confidence interval of the geometric mean ratio (GMR) of $\mathrm{AUC}$ and $\mathrm{C}_{\max }$ fall within $80-125 \%$ [1].

Progesterone is synthesized from a starting material from a plant source and is chemically identical to progesterone of human ovarian origin. Progesterone has a molecular weight of 314.47 and a molecular formula of $\mathrm{C}_{21} \mathrm{H}_{30} \mathrm{O}_{2}$. Progesterone (pregn-4-ene-3,20-dione) is a white or creamy white, odorless, crystalline powder practically insoluble in water, soluble in alcohol, acetone and dioxane and sparingly soluble in vegetable oils, stable in air, melting between $126^{\circ} \mathrm{C}$ and $131^{\circ} \mathrm{C}$. Progesterone Capsules are indicated in the treatment of Causing a period in premenopausal women with absent menstrual periods (secondary amenorrhea) and preventing abnormal overgrowth of the lining of the uterus (endometrial hyperplasia) in postmenopausal women taking estrogen hormone therapy [2].

Absorption: After oral administration of progesterone as a micronized soft-gelatin capsule formulation, maximum serum concentrations were attained within 3 hours. The absolute bioavailability of micronized progesterone is not known. Table 1 summarizes the mean pharmacokinetic parameters in postmenopausal women after five oral daily doses of Prometrium (Progesterone) Capsules $100 \mathrm{mg}$ as a micronized soft-gelatin capsule formulation [2].
Serum progesterone concentrations appeared linear and dose proportional following multiple dose administration of Prometrium (progesterone) Capsules $100 \mathrm{mg}$ over the dose range $100 \mathrm{mg} /$ day to 300 $\mathrm{mg} /$ day in postmenopausal women. Although doses greater than 300 $\mathrm{mg} /$ day were not studied in females, serum concentrations from a study in male volunteers appeared linear and dose proportional between 100 $\mathrm{mg} /$ day and $400 \mathrm{mg} /$ day. The pharmacokinetic parameters in male volunteers were generally consistent with those seen in postmenopausal women [2].

Distribution: Progesterone is approximately $96-99 \%$ bound to serum proteins, primarily to serum albumin (50-54\%) and transcortin (43-48\%) [2].

Excretion: The glucuronide and sulfate conjugates of pregnanediol and pregnanolone are excreted in the bile and urine. Progesterone

\begin{tabular}{|c|c|c|c|}
\hline \multirow{2}{*}{ Parameter } & \multicolumn{3}{|c|}{ Mean $\pm \mathrm{SD}$} \\
\cline { 2 - 4 } & $100 \mathrm{mg}$ & $200 \mathrm{mg}$ & $300 \mathrm{mg}$ \\
\hline $\mathrm{C}_{\max }(\mathrm{ng} / \mathrm{mL})$ & $17.3 \pm 21.9$ & $38.1 \pm 37.8$ & $60.6 \pm 72.5$ \\
\hline $\mathrm{T}_{\max }(\mathrm{hr})$ & $1.5 \pm 0.8$ & $2.3 \pm 1.4$ & $1.7 \pm 0.6$ \\
\hline AUC $_{(0-10)}\left(\mathrm{ng}{ }^{*} \mathrm{hr} / \mathrm{mL}\right)$ & $43.3 \pm 30.8$ & $101.2 \pm 66.0$ & $175.7 \pm 170.3$ \\
\hline
\end{tabular}

Table 1. Pharmacokinetic Parameters of Prometrium (progesterone) Capsules.

*Corresponding author: Someswara Rao K, Andhra University College of Pharmaceutical Sciences, Andhra University, Visakhapatnam, Visakhapatnam-530003, Tel: 91-9948591492; E-mail: korlas@rediff.com

Received May 19, 2014; Accepted July 28, 2014; Published August 04, 2014

Citation: Rajeswara Rao P, Someswara Rao K (2014) Intra Subject Variability of Progesterone $200 \mathrm{mg}$ Soft Capsules in Indian Healthy Adult Postmenopausal Female Subjects under Fasting Conditions. J Bioequiv Availab 6: 139-143. doi:10.4172/jbb.1000194

Copyright: @ 2014 Rajeswara Rao P, et al. This is an open-access article distributed under the terms of the Creative Commons Attribution License, which permits unrestricted use, distribution, and reproduction in any medium, provided the original author and source are credited. 
Citation: Rajeswara Rao P, Someswara Rao K (2014) Intra Subject Variability of Progesterone 200 mg Soft Capsules in Indian Healthy Adult Postmenopausal Female Subjects under Fasting Conditions. J Bioequiv Availab 6: 139-143. doi:10.4172/jbb.1000194

metabolites are eliminated mainly by the kidneys. Progesterone metabolites which are excreted in the bile may undergo enterohepatic recycling or may be excreted in the feces [2].

Food effect: Concomitant administration of progesterone capsules with food increased the bioavailability of progesterone capsules relative to a fasting state when administered to postmenopausal women at a dose of $200 \mathrm{mg}$ [2]. Progesterone is a steroid hormone which plays an important role in the preparation and maintenance of pregnancy. Under its influence the numerous minute glands line the uterine cavity are transformed into secreting glands. This alteration is a part of the change which is essential to provide for the implantation of a fertilized ovum and for the continuing development of the placenta. Progesterone is synthesized from cholesterol via pregnenalone, and then rapidly metabolized to pregnanediol, for the most part in the liver. The ovary and placenta are major production sites, but a small amount is also synthesized by the adrenal cortex in both men and women ${ }^{3}$.

Circulating progesterone levels which are characteristically low during the follicular phase $(<1.5 \mathrm{ng} / \mathrm{mL})$, increase sharply during the luteal phase of the menstrual cycle, reaching a maximum ( $>20 \mathrm{ng} /$ $\mathrm{mL}$ ) some 5 to 10 days after the mid cycle LH peak. Unless pregnancy occurs, a steep decline to follicular level sets in about 4 days prior to the next menstrual period [3].

The biologic effects of progesterone may be grouped as follows: (1) uterine endometrial cells are transformed in such a way that they may receive the early embryo and facilitate its implantation; (2) myometric activity is suppressed, aiding in retention of the embryo during implantation and growth prior to normal parturition; (3) numerous and varied metabolic parameters may be altered which may have no direct impact on maintenance and termination of pregnancy [3].The aim of this study was to identify intra subject variability of progesterone soft capsules, to compare the rate and extent of absorption of Progesterone USP capsules $200 \mathrm{mg}$ (Test) manufactured by Strides Arcolab Limited and Prometrium ${ }^{\circ}$ (Progesterone USP) capsules $200 \mathrm{mg}$ (Reference) marketed by Solvay Pharmaceuticals Inc., Marietta, GA when given in equal doses of single oral dose in 12 healthy, human, post menopausal female subjects under fasting conditions and to monitor the adverse events and to ensure the safety of the subjects.

\section{Material And Methods}

The tested treatment details are as given below for the conducted study.

\section{Reference product $(\mathbf{R})$}

Prometrium ${ }^{\star}$ (Progesterone USP) capsules $200 \mathrm{mg}$; each capsule contains $200 \mathrm{mg}$ of progesterone micronized, USP, Marketed by Solvay Pharmaceuticals Inc., Marietta, GA.

Batch No: 510299

Exp. Date: 10/2009

\section{Test product $(\mathrm{T})$}

Progesterone Usp Capsules 200 mg; each capsule containing 200 mg of progesterone Micronized, USP, Manufactured By Aurobindo Pharma Limited, India.

Batch No: 7204798

Mfg. Date: 10/2007
Exp. Date: 09/2009

\section{EXP. DATE: 09/2009}

\section{Study design}

This study was an open label, randomized, balanced, singledose, two sequence, two period, crossover oral bioequivalence study was conducted in 12 healthy adult, human, post menopausal female volunteers under fasting conditions. All the subjects provided written informed consent to participate in the study prior to enrolment and were free to withdraw at any time during the study. The study was conducted in compliance with the ICH GCP, ICMR guidelines, and declaration of Helsinki at the research facility.

\section{Screening}

Volunteers aged from 40-55 years with a body mass index (BMI) in the range of $18-29.9 \mathrm{~kg} / \mathrm{m}^{2}$ were selected according to the inclusion and exclusion criteria. They were assessed to be healthy according to medical, systemic and physical examination including vital signs, and normal laboratory test results (haematology, biochemistry, urine analysis), Follicle stimulating hormone (FSH), Prothrombin time (PT), Activated Partial Thromboplastin Time (APTT), Estradiol, Papanicolaou smear, Mammogram, Ultra sound pelvis, 12-lead ECG, Chest X-ray (PA view) and Screening for infectious diseases including negative HIV-1 \& -2, Hepatitis B, Hepatitis C, RPR tests.

Drugs of abuse (Benzodiazepines, Opioids, Amphetamines, Cannabinoids, Cocaines and Barbiturates) in urine, Urine Pregnancy test and alcohol breath analysis test were performed during the study check-in of each period and who tested negative were checked-in.

\section{Drug administration}

After an over night fasting of at least 10 hours a single oral dose of Progesterone USP capsules $200 \mathrm{mg}$, Test (T) or Reference (R) product were administered in sitting posture as per randomization schedule with $240 \mathrm{~mL}$ of drinking water at room temperature under fasting condtions.

\section{Blood sampling schedule}

A total of 21 blood samples ( $4 \mathrm{~mL}$ each) in each period were collected in a pre-labeled vacutainer tubes containing K EDTA. The blood samples were withdrawn pre-dose at $24.00,0.00$ hours and at $0.33,0.67,1.00,1.50,2.00,2.50,3.00,3.50,4.00,4.50,5.00,5.50,6.00$, $8.00,10.00,12.00,18.0024 .00$ and 36.0 hours post dose in each period. The collected samples were centrifuged and separated plasma samples were transferred into pre labeled polypropylene tubes as single aliquot and were stored in a deep freezer maintained at $-80^{\circ} \mathrm{C}$ or colder until Bio-analysis.

\section{Washout period}

A period of ten (10) days was given between the any two periods

\section{Study conduct}

A total of 12 subjects were enrolled in the study. All the 12 subjects were completed all the periods of the crossover.

\section{Analytical method}

Progesterone was analyzed using validated LC-MS/MS method. The run time is 3.4 minutes, polarity: +ve mode, column used is Zodaic Sil 120-3-C18, AQ $3.0 \mu 4.6 \times 100$. Mobile Phase: $2 \mathrm{mM}$ Ammonium Formate ( $\mathrm{pH}$ 6.2): Methanol: Acetonitrile $(\mathrm{ACN})$ in the ratio of 
Citation: Rajeswara Rao P, Someswara Rao K (2014) Intra Subject Variability of Progesterone 200 mg Soft Capsules in Indian Healthy Adult Postmenopausal Female Subjects under Fasting Conditions. J Bioequiv Availab 6: 139-143. doi:10.4172/jbb.1000194

10:20:70. Flow rate was $1.0 \mathrm{~mL}$, injection volume is $10 \mathrm{~mL}$, retention time for progesterone is 2.55 and for progesterone $\mathrm{d} 9$ is 2.50 .

Fifty mililiter of internal standard was taken $(500 \mathrm{ng} / \mathrm{mL})$ which was mixed with $0.4 \mathrm{~mL}$ of plasma and then $0.4 \mathrm{~mL} 2 \%$ OPA solution was added and vertexed for $10 \mathrm{~min}$. Extracted the solution with Solid Phase Extraction using HCB Barry/ICC and conditioned with $1 \mathrm{~mL}$ methanol and $1 \mathrm{~mL}$ of milliQ water. The plasma sample was loaded and washed with $1 \mathrm{~mL}$ of $0.2 \%$ ammonia solution. Then the obtained solution was washed with $1 \mathrm{~mL}$ of $10 \%$ methanol and then the cartridges dried for 2 minutes. The solution was eluted with $1 \mathrm{~mL}$ of Acetonitrile $(\mathrm{ACN})$ and evaporated for 10 minutes and finally reconstituted with $0.4 \mathrm{~mL}$ mobile phase and injected the sample on LC/MS/MS System. The calibration curve range used is $1.00 \mathrm{ng} / \mathrm{mL}$ to $80.00 \mathrm{ng} / \mathrm{mL}$.

\begin{tabular}{|c|c|c|}
\hline & Q1 & Q3 \\
\hline Progesterone & 315.5 & 97.3 \\
\hline Progesterone d9 & 324.5 & 100.3 \\
\hline
\end{tabular}

\section{Pharmacokinetic and statistical analysis}

Calculation of pharmacokinetic parameters was done for progesterone baseline-corrected data and progesterone baselineuncorrected data using drug concentration time data by noncompartmental method using WinNonlin professional software version 5.0.1 (Pharsight Corporation, USA). Statistical analysis of the pharmacokinetic parameters of the two formulations was carried out using PROC GLM of SAS release 9.1.3 (SAS Institute Inc., USA) to assess the comparative oral bioavailability of progesterone baselinecorrected data and progesterone baseline-uncorrected data. Descriptive statistics, ANOVA, nominal 90\% confidence intervals, intra-subject variability of reference product was computed and reported for primary and secondary pharmacokinetic parameters of progesterone baselinecorrected data and progesterone baseline-uncorrected data [4].

Pharmacokinetic parameters of the formulations, based on the following primary and secondary pharmacokinetic parameters were assessed:

For Progesterone baseline-corrected data the following pharmacokinetic parameters were estimated.

$\mathbf{C}_{\max }$ : Maximum measured plasma concentration over the time span specified.

AUC $_{0-\mathrm{t}}$ : The area under the plasma concentration versus time curve, from time 0 to the last measurable concentration, as calculated by the linear trapezoidal method.

AUC $_{0-\infty}$ : The area under the plasma concentration versus time curve from time 0 to time infinity.

$$
\text { AUC }_{\% \text { Extrapolation }}:\left(\left(\mathrm{AUC}_{0-\infty}-\mathrm{AUC}_{0-\mathrm{t}}\right) / \mathrm{AUC}_{0-\infty}\right) \times 100
$$

$\mathbf{T}_{\max }:$ Time of the maximum measured plasma concentration. If the maximum value occurs at more than one time point, $\mathrm{T}_{\max }$ is defined as the first time point with this value.

$\mathbf{K}_{\mathrm{el}}$ : Apparent first order elimination rate constant calculated from a semi-log plot of plasma concentration versus time point. The parameter was calculated by linear square regression analysis using the last 3 (or more) non-zero plasma concentrations. $\mathrm{K}_{\mathrm{el}}$.

$\mathbf{T}_{1 / 2}$ : The elimination or terminal half-life was calculated as 0.693 /

For Progesterone baseline-uncorrected data the following pharmacokinetic parameters were estimated.
$\mathbf{C}_{\max }$ : Maximum measured plasma concentration over the time span specified.

$\mathbf{A U C}_{\mathbf{0} \mathrm{t}}$ : The area under the plasma concentration versus time curve, from time 0 to the last measurable concentration, as calculated by the linear trapezoidal method.

$\mathbf{T}_{\max }$ : Time of the maximum measured plasma concentration. If the maximum value occurs at more than one time point, $\mathrm{T}_{\max }$ is defined as the first time point with this value.

These parameters were derived individually for each analyzed subject from the concentration vs. time data of Progesterone baseline-corrected and Progesterone baseline-uncorrected in plasma. Values below the lower limit of quantification were set to zero. The pharmacokinetic parameters were calculated by non-compartmental model using WinNonlin Professional Software Version-5.0.1 (Pharsight Corporation, USA).

\section{Results And Discussion}

The descriptive statistics, ANOVA, nominal 90\% confidence intervals, intra-subject variabilty were computed for the pharmacokinetic parameters of progesterone baseline-corrected data were as mentioned below (Table 2).

The log-transformed pharmacokinetic parameters, $\mathrm{C}_{\max }, \mathrm{AUC}_{0-\mathrm{f}}$ and $\mathrm{AUC}_{0-\infty}$ of baseline corrected data for progesterone were subjected to analysis of variance (ANOVA) with the main effects of sequence, treatment, and period at $5 \%$ level of significance. The obtained intrasubject variabilty for $\mathrm{C}_{\max }, \mathrm{AUC}_{0-\mathrm{t}}$ and $\mathrm{AUC}_{0-\infty}$ were found to be $68.2 \%$, $75.6 \%$ and $64.6 \%$ respectively. The obtained geometric least squares means for test product of $\mathrm{C}_{\max }, \mathrm{AUC}_{0-\mathrm{t}}$ and $\mathrm{AUC}_{0-\infty}$ were found to be $16.1833,52.5182$ and 55.4791 respectively. The obtained geometric least squares means for reference product of $\mathrm{C}_{\max }, \mathrm{AUC}_{0-\mathrm{t}}$ and $\mathrm{AUC}$ $\infty$ were found to be $18.3805,56.3681$ and 59.9745 . The T/R ratio for were found to be $88.05 \%, 93.17 \%$ and $92.50 \%$ respectively. The $90 \%$ confidence intervals for $\mathrm{C}_{\max }, \mathrm{AUC}_{0 \text {-t }}$ and $\mathrm{AUC}_{0-\infty}$ using nominal average bioequivalence approach were found to be $52.10-148.80 \%$, $52.66-164.84 \%$ and $56.05-152.68 \%$ respectively. The obtained nominal $90 \%$ confidence intervals were fell outside the acceptance range of 80.00-125.00\% (Figure 1-4).

\section{Conclusions}

Based on the results obtained using average bioequivalence criterian,

\begin{tabular}{|c|c|c|c|c|c|}
\hline \multirow{2}{*}{\multicolumn{2}{|c|}{ Parameter (Unit) }} & \multicolumn{4}{|c|}{$\begin{array}{c}\text { Mean } \pm \text { SD } \\
\text { (Un-transformed data) } \\
\text { Progesterone baseline-corrected }\end{array}$} \\
\hline & & \multicolumn{2}{|c|}{ Test Product (T) } & \multicolumn{2}{|c|}{ Reference Product (R) } \\
\hline \multicolumn{2}{|c|}{$\mathrm{C}_{\max }(\mathrm{ng} / \mathrm{mL})$} & \multicolumn{2}{|c|}{$21.2850 \pm 13.55611$} & \multicolumn{2}{|c|}{$19.5116 \pm 10.04936$} \\
\hline \multicolumn{2}{|c|}{$A \cup C_{0 \rightarrow t}(\mathrm{hr} . \mathrm{ng} / \mathrm{mL})$} & \multicolumn{2}{|c|}{$68.3649 \pm 51.62570$} & \multicolumn{2}{|c|}{$70.0821 \pm 57.54212$} \\
\hline \multicolumn{2}{|c|}{$\mathrm{AUC}_{0 \rightarrow \infty}(\mathrm{hr} . \mathrm{ng} / \mathrm{mL})$} & \multicolumn{2}{|c|}{$70.5905 \pm 51.74586$} & \multicolumn{2}{|c|}{$73.0414 \pm 57.78191$} \\
\hline \multicolumn{2}{|l|}{$\mathrm{T}_{\max }(\mathrm{hr})$} & \multicolumn{2}{|c|}{$1.50(1.50-5.00)$} & \multicolumn{2}{|c|}{$2.50(1.00-4.00)$} \\
\hline \multicolumn{2}{|l|}{$\mathrm{K}_{\mathrm{el}}\left(\mathrm{hr}^{-1}\right)$} & \multicolumn{2}{|c|}{$0.19783 \pm 0.143029$} & \multicolumn{2}{|c|}{$0.10196 \pm 0.102788$} \\
\hline \multicolumn{2}{|l|}{$t_{1 / 2}(h r)$} & \multicolumn{2}{|c|}{$7.516 \pm 10.3630$} & \multicolumn{2}{|c|}{$11.508 \pm 6.9087$} \\
\hline \multirow{3}{*}{ Parameters } & \multicolumn{5}{|c|}{ Untransformed Data } \\
\hline & \multicolumn{2}{|c|}{ Geometric Mean } & \multirow{2}{*}{$\begin{array}{l}\text { (T/R) } \\
\text { Ratio } \\
\text { (\%) }\end{array}$} & \multirow{2}{*}{$\begin{array}{l}90 \% \\
\text { Confidence } \\
\text { Interval }\end{array}$} & \multirow{2}{*}{$\begin{array}{l}\text { Intra } \\
\text { Subject } \\
\text { CV (\%) }\end{array}$} \\
\hline & $\begin{array}{l}\text { Test } \\
\text { (T) }\end{array}$ & $\begin{array}{l}\text { Reference } \\
\text { (R) }\end{array}$ & & & \\
\hline $\mathbf{C}_{\max }$ & 16.1833 & 18.3805 & 88.05 & $52.10-148.80$ & 68.2 \\
\hline $\mathrm{AUC}_{0 \rightarrow \mathrm{t}}$ & 52.5182 & 56.3681 & 93.17 & $52.66-164.84$ & 75.6 \\
\hline $\mathrm{AUC}_{0 \rightarrow \infty}$ & 55.4791 & 59.9745 & 92.50 & $56.05-152.68$ & 64.6 \\
\hline
\end{tabular}


Citation: Rajeswara Rao P, Someswara Rao K (2014) Intra Subject Variability of Progesterone 200 mg Soft Capsules in Indian Healthy Adult Postmenopausal Female Subjects under Fasting Conditions. J Bioequiv Availab 6: 139-143. doi:10.4172/jbb.1000194

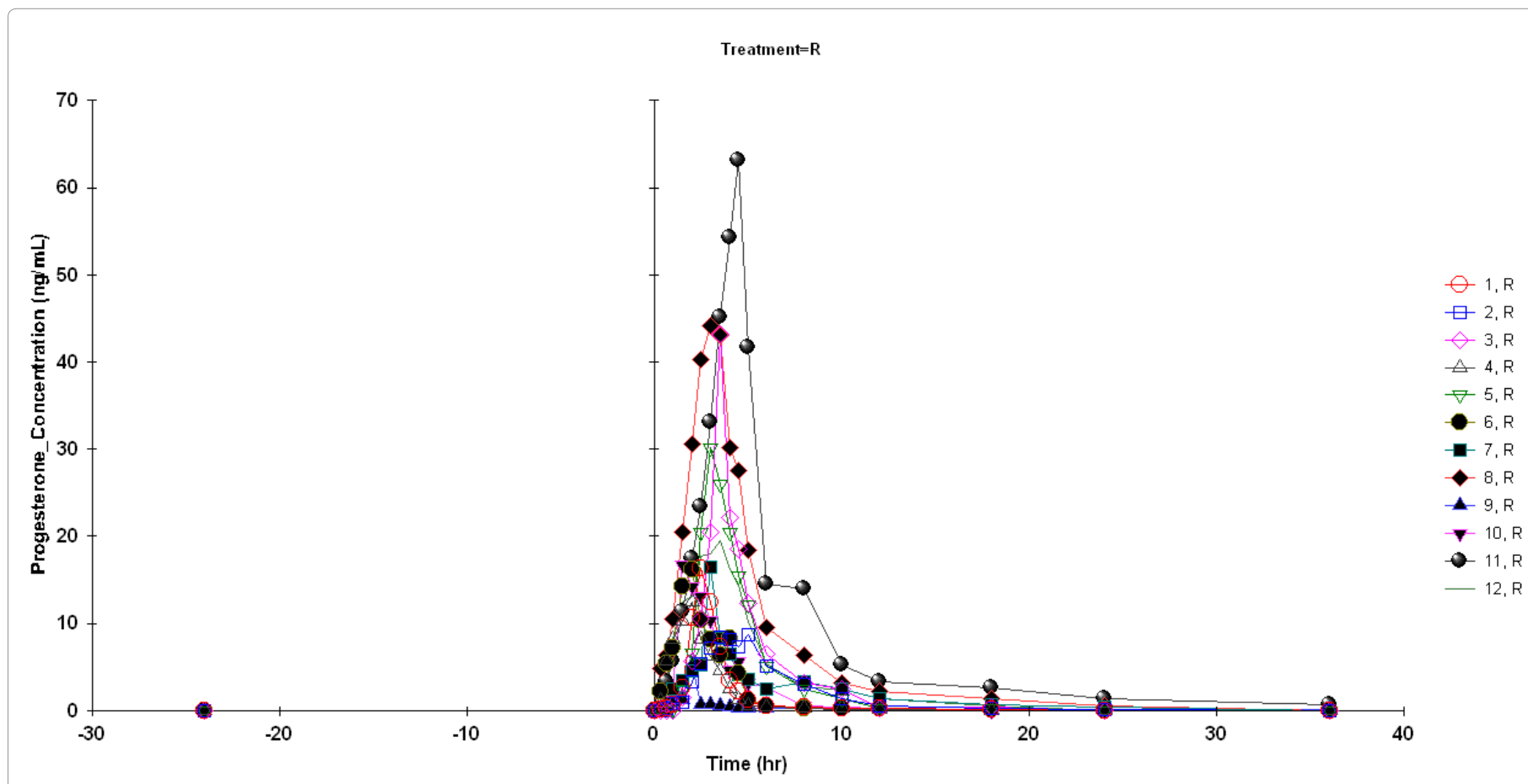

Figure 1: Plasma Concentration versus Time Profile of Progesterone (ng/mL) Under Fasting Conditions for all 12 subjects in linear scale.

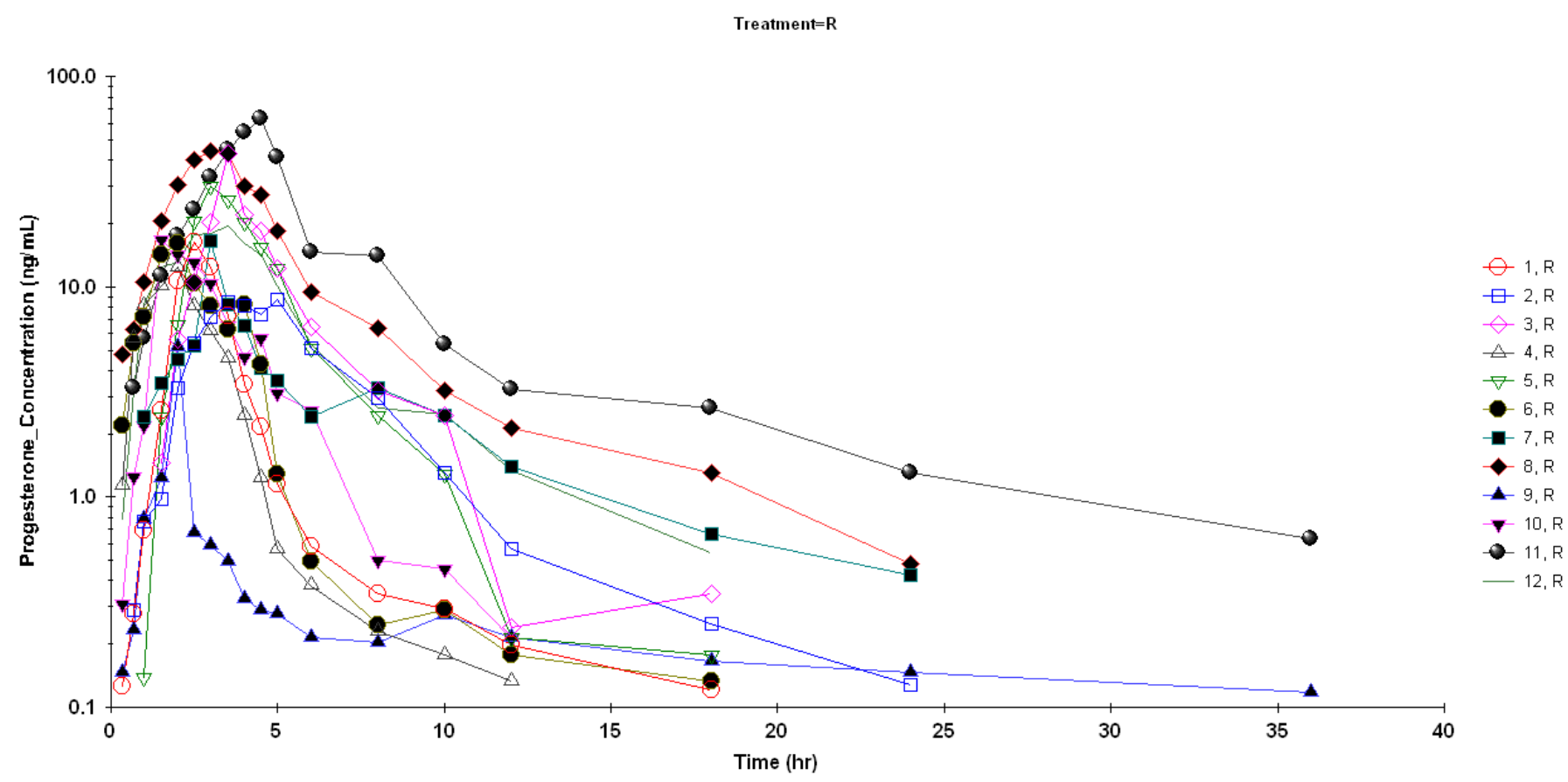

Figure 2: Semi-log Plasma Concentration versus Time Profile of Progesterone ( $\mathrm{ng} / \mathrm{mL})$ Under Fasting Conditions for all 12 subjects in linear scale.

the test product fails to prove the bioequivalence in comparison with that of innovator. The global variance and the within subjet standard deviation of reference product was greater than at least $60 \%$ for Cmax,
$\mathrm{AUC}_{0-\mathrm{t}}$ and $\mathrm{AUC}_{0-\infty}$ under fasting conditions. Based on these results it has been concluded that oral progesterone was found to be highly variable and exhibits erratic abosrption pattern from the formalation. 
Citation: Rajeswara Rao P, Someswara Rao K (2014) Intra Subject Variability of Progesterone 200 mg Soft Capsules in Indian Healthy Adult Postmenopausal Female Subjects under Fasting Conditions. J Bioequiv Availab 6: 139-143. doi:10.4172/jbb.1000194

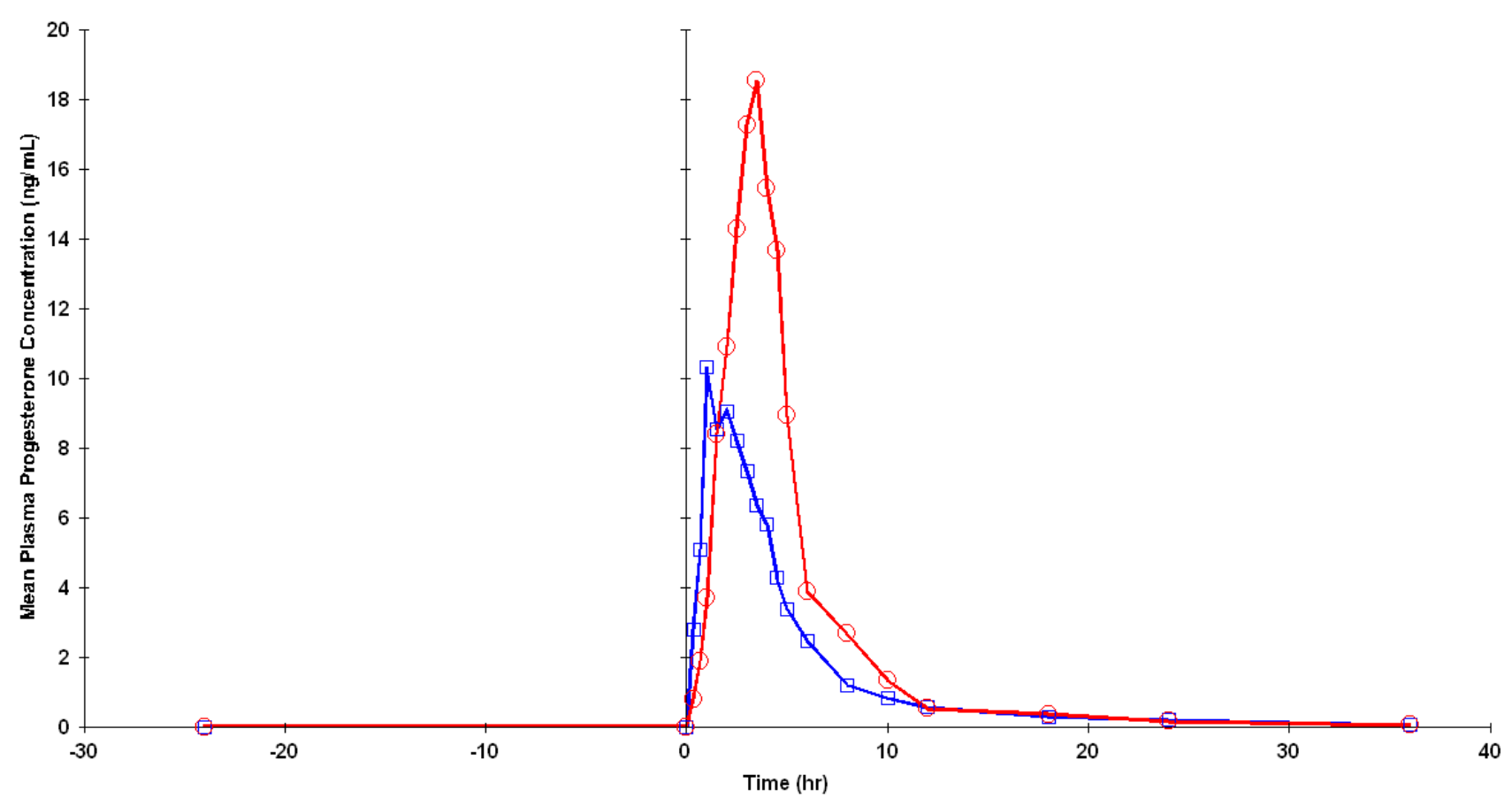

Figure 3: Mean Plasma Concentration versus Time Profile of Progesterone (ng/mL) Under Fasting Conditions for all 12 subjects in linear scale.

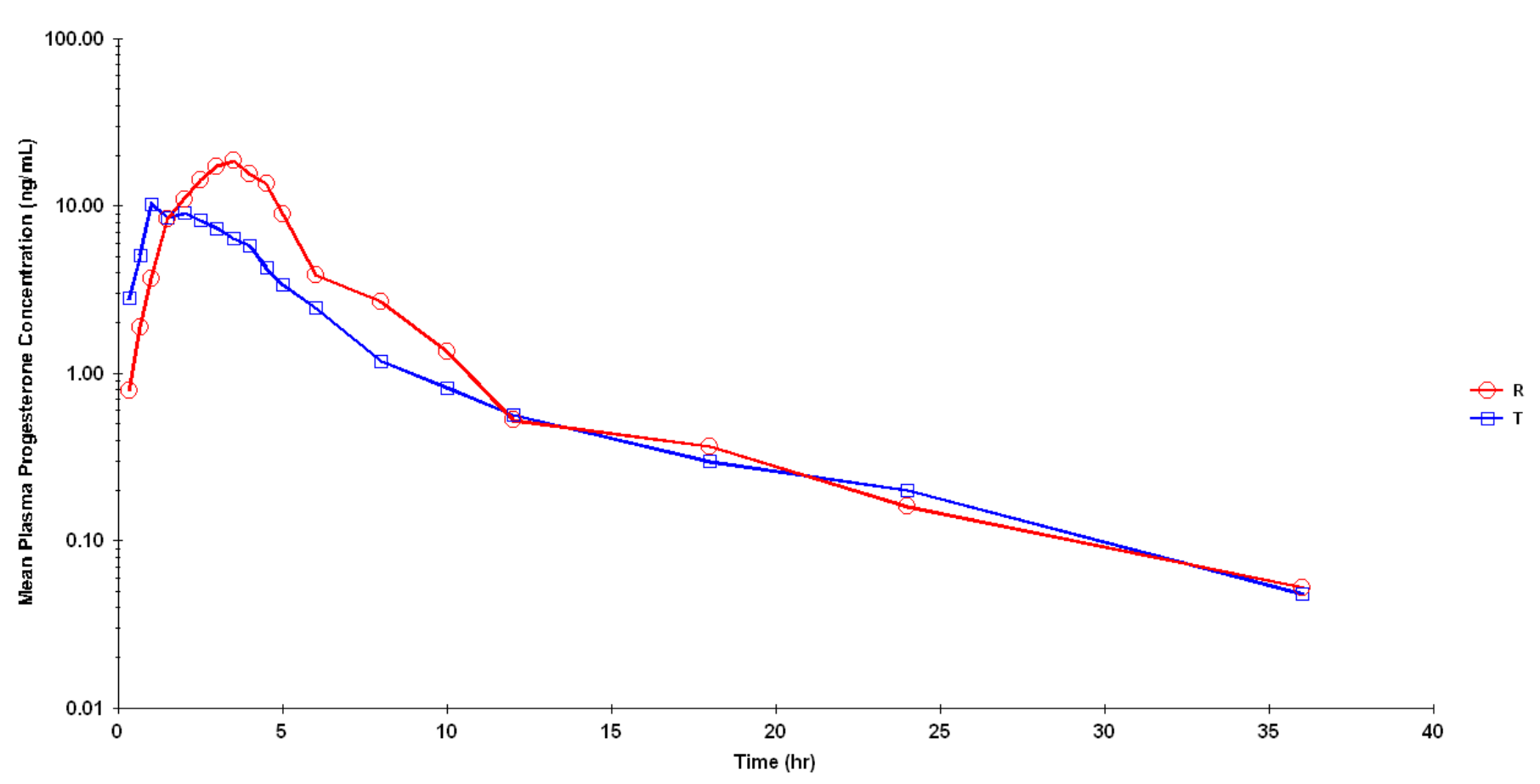

Figure 4: Semi-log Mean Plasma Concentration versus Time Profile of Progesterone (ng/mL) Under Fasting Conditions for all 12 subjects.

Baseline values are also not detected indicated that a sensitive LOQ of $5 \mathrm{pg} / \mathrm{mL}$ is required for appropriate detection baseline concentrations.

\section{References}

1. U.S. Food and Drug Administration. Guidance for Industry.
2. (2003) Bioavailability and Bioequivalence Studies for Orally Administered D drug Products-General Considerations.

3. (2011) Prescribing Information of Prometrium 1-26.

4. Paul Back (1995) Metabolic Effects of Gonadal Hormones and Contraceptive Steroids. pp 339-351. 